\title{
Scaling Properties of Silicon Microring CROWs (Project Report 0925399-Y3)
}

\author{
SHAYAN MOOKHERJEA \\ Department of Electrical and Computer Engineering, University of California, San Diego, MC 0407 La \\ Jolla CA 92093-0407 USA \\ Email:smookher@ucsd.edu
}

The research outcomes of this project include the realization of long silicon coupled-resonator optical waveguides and a study of their optical transmission and localization properties.

\section{Introduction and Goals}

The goal of this NSF-funded project [0925399] is to design, fabricate and study a new class of photonic circuits and devices in which disorder can be harnessed and controlled. We used the coupled-resonator optical waveguide (CROW) device structure as the foundation of this project because although tight-binding models of propagation exist [1]-[4], optical propagation near the band-edge regions which have high dispersion and high nonlinearity [5] are not easy to calculate and these regimes are highly sensitive to disorder [6], [7]. There is also a fundamental reason to develop our understanding of nonlinear optics, since the optical Kerr nonlinearity which is used as the physical principle for four-wave mixing is important in other topics of nonlinear optics such as the study of bright and dark solitons [8], [9] which can also be observed in coupledresonator systems [10]. Measurements made in this project were compared to earlier theoretical models [11]-[13]. CROWs can have many potential applications including low-power nonlinear optics [14]-[16], novel lasers [17] and generation of entangled photon pairs through spontaneous four-wave mixing (SFWM) [18]. Longer CROWs can be helpful in nonlinear optics [14] and quantum photonics [19]. In this project, we firstly intend to develop scientific insights in designing optical structures which utilize disorder for functionality. Secondly, we plan to make improvements in methods for correcting or compensating for defective order where needed.

\section{Activities}

During the third year (2011-12) of this project, we have developed a hybrid theoretical and computational approach to answer the question: Given a certain level of disorder, what is the maximum length of the coupled-resonator chain that still supports propagation? For the first time, we obtained a formula that describes how rapidly the bandwidth of a CROW drops to zero, as disorder effects build up over the propagation length. The bandwidth collapse is nonlinear and is a critical phenomenon, which occurs abruptly once a certain 
threshold is exceeded. Conventionally, the approach to maximizing the delay capacity of a slow-light waveguide was based on minimizing the coupling between adjacent resonators (i.e., making a small number of very-high-Q resonators), but this turns out to be true only when disorder effects are ignored. Better performance, instead, can be achieved by making a larger number of relatively modest-Q resonators since the effects of disorder are manifest less severely in longer chains of coupled resonators.

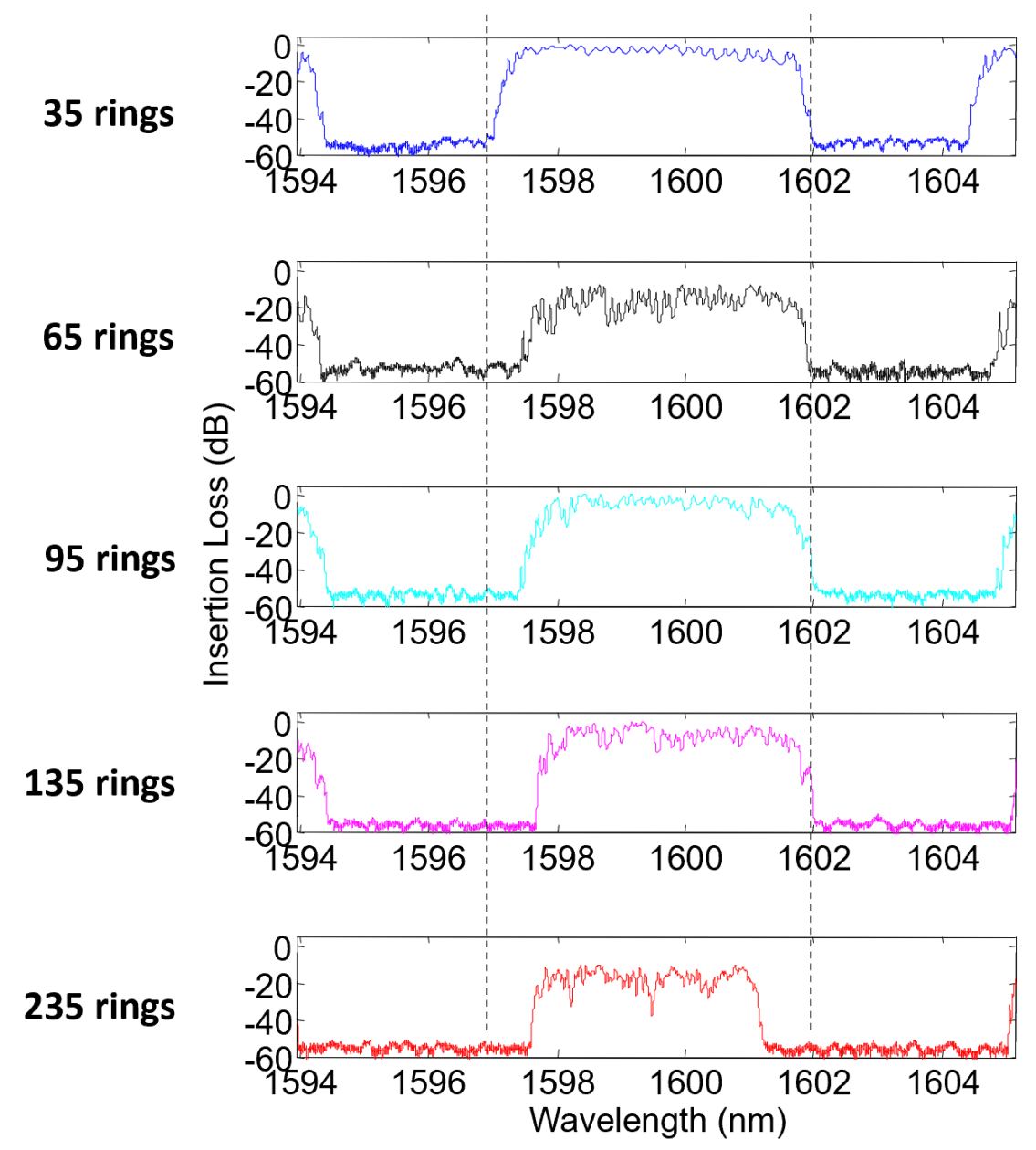

Figure 1 Gradual band-narrowing, increasing loss, and increasing transmission (intensity) ripple in CROWs with increasing length. These measurements were performed on silicon CROWs with 35 to 235 coupled microring resonators in the racetrack configuration. Here, transmission of a single passband is shown for each device; similar measurements were performed for other passbands across the $\mathrm{C}$ and $\mathrm{L}$ wavelength bands between $1520 \mathrm{~nm}$ and $1610 \mathrm{~nm}$.

Transmission measurements on CROW structures are shown in Fig. 1. These CROW structures are based on silicon microring resonators with directional couplers. Previously, we have experimentally studied the dispersion of such couplers using a microring coupled to a waveguide [20] and also studied the dispersion relationship of a CROW theoretically [13]. An accurate knowledge 
of dispersion is also necessary for planar lightwave circuits which use CROWs (as filters) together with other devices [21]. We developed an extension of coupled-mode theory which is applicable to directional couplers in silicon photonics, coupled-waveguide structures [22] as well as coupled-resonator structures [23]. CROWs of up to 235 coupled resonators were successfully fabricated and measured, and a good agreement was achieved between experiments and theory [24]-[26].

A more detailed understanding of waveguiding in CROWs can be obtained through a detailed analysis of the statistical properties of the transmission and propagation time. Such measurements were carried out in the optical domain for the first time in our work (see Figure 2). The distinction between normal, non-localized transport and near-localization transport was clearly seen through the statistics of the normalized intensity, and the normalized group delay.

a
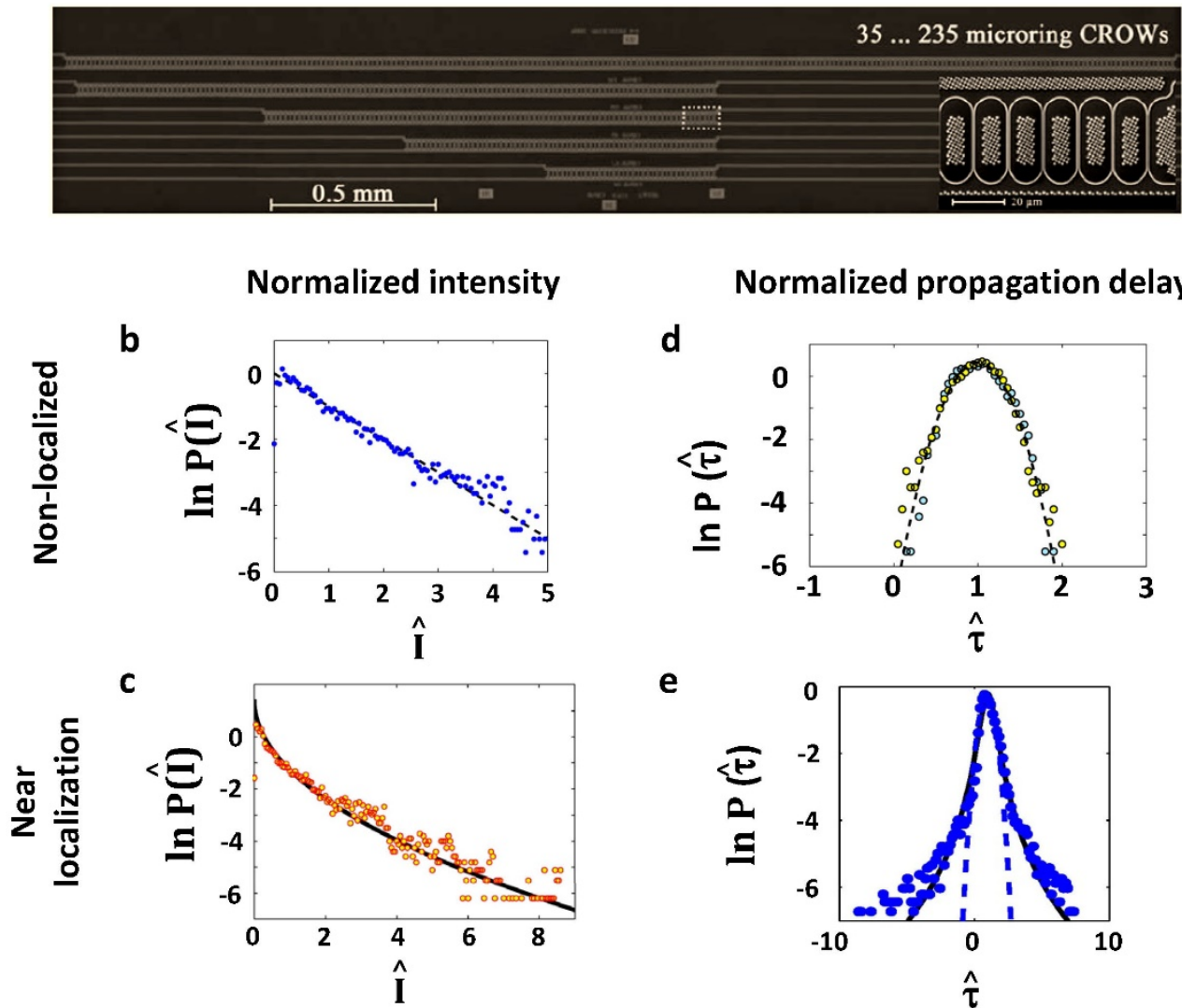

Normalized propagation delay

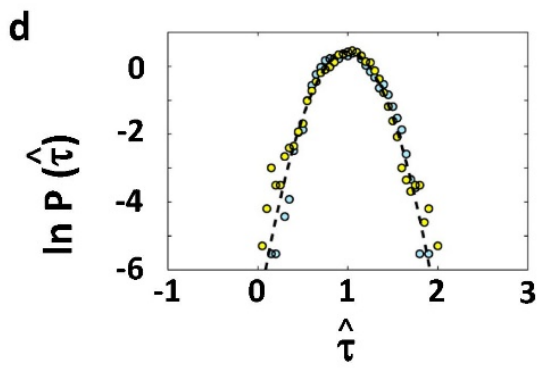

e

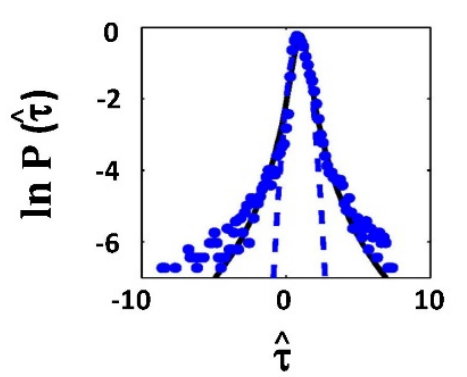

Figure 2 (a), Optical microscope image of a cluster of coupled-resonator optical waveguides, upto 235 rings in length. (b), (c), Statistical distributions of normalized transmitted intensity for non-localized and near-localized light in different chips, showing agreement with, and deviation from, the Rayleigh distribution, respectively. (d), (e), Statistical distributions of normalized propagation group delay for non-localized and near-localized light, showing agreement with, and deviation from, a Gaussian distribution. The solid line in Fig. 1(e) is a distribution with polynomial tails, with exponent $-3 / 2$. 
The scaling of the normalized propagation delay can be studied in more detail, by subtracting out the mean value, and examining the statistics of the residual quantity, which is called the group-delay ripple (GDR). As shown in Figure 3, the band-center GDR scales quadratically with waveguide length in the case of the conventional waveguides, but scales in an interesting way in CROWs. Although GDR increases for longer CROWs, the rate of increase decreases. Theory shows that as N, the number of unit cells increases, the GDR should asymptotically reach a constant value. In contrast, the GDR of conventional silicon waveguides (like any uncorrelated random walk process) continues to increase sharply with length. This difference is attributed a known physical phenomenon called "exchange narrowing" in coupled-oscillator systems, in which the disorder strength (potential) is renormalized by the number of coupled resonators, and hence becomes relatively weaker in longer chains of correlated oscillators.

However, in absolute terms, the GDR of CROWs is nearly an order of magnitude larger than that of low-loss silicon waveguides. Therefore, although the screening of disorder in CROWs offers interesting physics, the overall performance of CROWs will have to be reduced in order for better performance compared to simple waveguides in practical applications as optical delay lines. This is shown in Figure 4. However, CROWs with the relatively low perresonator loss and low GDR that was achieved outperformed conventional waveguides in nonlinear optics applications such as four-wave mixing and entangled photon-pair generation.

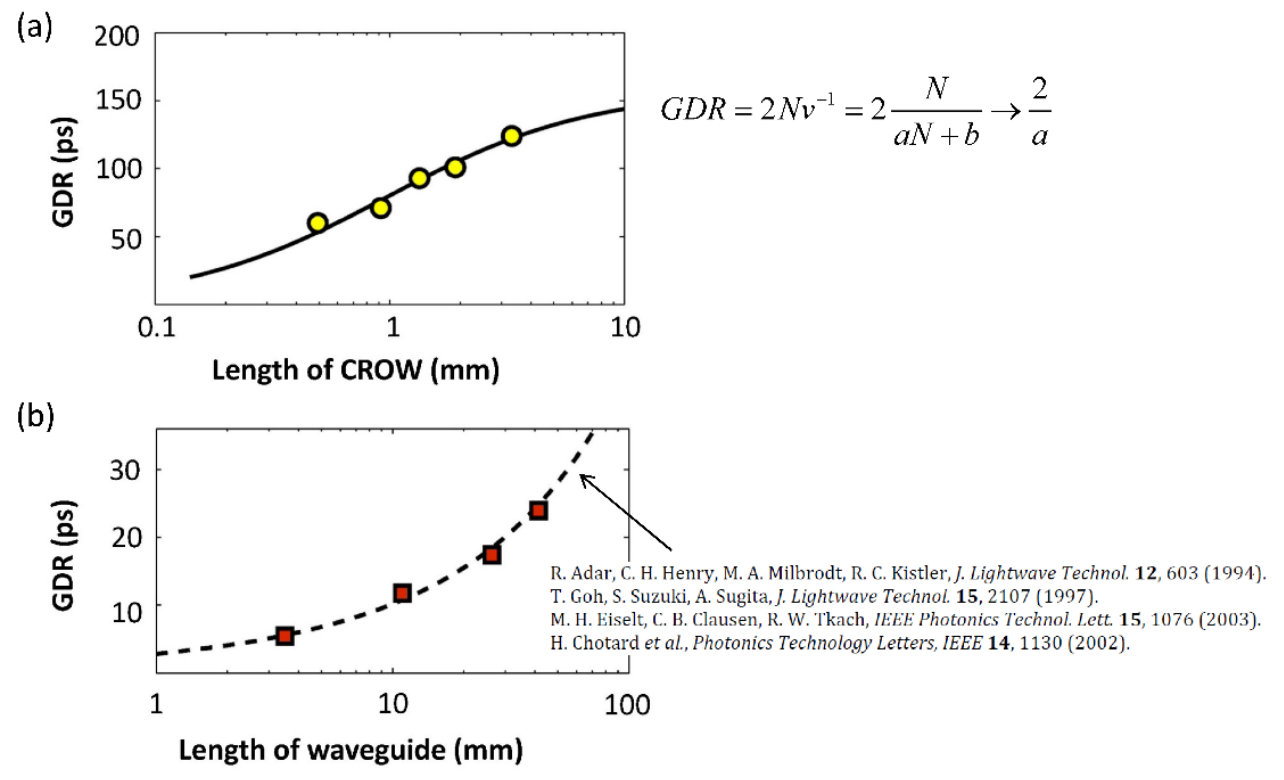

Figure 3 (a) Scaling of group delay ripple (GDR) versus CROW length (experimental data points shown in yellow circles). The solid line is obtained from simulations and is not a fit to the data. 
As the number of unit cells (N) increases, GDR tends to a constant. (b) GDR versus length for conventional silicon waveguides, which exhibits quadratically increasing scaling with length.

\section{Progress and Impact}

The CROWs shown in Figure 2(a) are passive devices. It is useful to incorporate a $\mathrm{p}-\mathrm{i}-\mathrm{n}$ diode across a waveguide cross-section as shown using a microring resonator for four-wave mixing [27]. We require an external control "knob" to tune the waveguiding properties of the CROW structure. We decided to use electro-optic tunability for most of our studies. Using electro-optic control fields was practically convenient since it was easy to generate the switching waveforms in the laboratory [28]. If tuning speed is not a concern, the thermo-optic effect, which we used for tunable filters, is also another option as a control knob [29] as is optical gain [30]. Alternatively, optical pump beams can be used to manipulate the waveguiding properties through nonlinear optical properties. This can be difficult to control in practice since silicon microresonators can be affected by some phenomena that are not always desirable for CROWs, such as bistability [31]. Infrared imaging is a useful experimental tool to study light propagation through the length of these structures [32], [33].

We reported the first demonstration of single photon generation in a siliconbased photonic device. This was a CROW waveguide which achieves high performance in a particular type of nonlinear optical wave-mixing phenomenon (spontaneous four-wave mixing, SFWM) based on careful attention to realistic disorder and other non-ideality effects. This CMOS-compatible device uses only silicon and silicon dioxide as constituent materials, operates at room temperature and in the technologically-important telecommunications wavelength band, with all wavelengths (including the pump for SFWM) near $1550 \mathrm{~nm}$.

We first generated photon pairs through SFWM, as had recently been shown by a few groups. However, photon pair generation by itself is not sufficient for demonstrating single photon generation. Instead, one must implement heralding, a process by which one photon of the pair is detected and projects the system into a single photon Fock state, whose character must then be confirmed through a photon correlation measurement. We performed these measurements and demonstrate photon antibunching of the heralded signal $\left[g^{(2)}(0)=0.19\right]$, an unambiguous signature of single photon generation.

External collaborations: A collaboration with IBM's silicon photonics group (Dr. W. M. J. Green) provided insights into silicon photonics fabrication and also supported technical collaboration on fabricating these structures. Several 
jointly-authored publications resulted from this collaboration [12], [34], [35]. Dr. Ivan B. Divliansky (Research Scientist, CREOL, Florida) collaborated with us for the electron-beam lithography of long waveguide structures. Jointlyauthored publications resulted from this collaboration [32].

Novel measurement techniques were developed based on a single-scan, Jones matrix-based, interferometric spectral measurement instrument (Luna Tech. Optical Vector Analyzer OVA5000, generously loaned by Luna Technologies, VA. A jointly-authored journal article was published in Optics Letters from this collaboration [12].

Impact on Human Resources: Three students in the PI's group (one supported directly by this grant, one primarily supported by the PI's CAREER grant, and the third by an NSF Graduate Fellowship) have worked on this topic during the past year as part of their education and training. A post-doctoral scholar participated in this research and presented results at an invited talk at the SPIE Photonics West 2010 conference. Three undergraduates participated in summer research activities related to this project. One of the undergraduates was a Regents Scholar at UCSD and continued his research during the academic year.

Students have been trained on several aspects of scientific research including:

- theoretical calculations, using our whole-domain spectral representation of CROWs developed by two graduate students

- numerical simulations using standard software packages as well as an inhouse development effort for our own finite-difference frequency-domain vectorial field solver, written primarily by a graduate student,

- device fabrication (including electron-beam lithography, reactive ion etching, wafer lapping and cleaving, AFM metrology, etc.) and a novel form of device tuning based on AFM nano-oxidation developed by a graduate student - optical device measurement (including use of tunable lasers, optical spectrum analyzers, fiber-waveguide coupling)

- presentation of research results, including co-authoring of scientific papers and conference publications.

\section{Subsequent Extensions}

The physics of disordered chains is rich and full of useful insights for device engineers [36]-[38]. As mentioned earlier, some of the most interesting physics of CROWs, such as exchange narrowing and disorder screening, can be of practice utility only if the overall level of disorder in the fabricated device can be reduced so that important parameters such as loss and group delay ripple (GDR) are at least comparable to that of conventional silicon waveguides. This is made clear by the comparison in Figure 4. The most common effect of 
disorder is that the resonance frequency of a microring resonator is randomly shifted by hundreds of gigahertz because of few-nanometer variations in the width or height, commonly encountered during device fabrication, and these errors can cause phase fluctuations which affect the CROW transmission properties, especially group delay (Mach-Zehnder modulators are also affected [39]). If nanoscale disorder can be modified, device performance may be improved.

\begin{tabular}{|l|l|}
\hline Silicon waveguide & Coupled resonator waveguide \\
\hline Loss $=1 \mathrm{~dB} / \mathrm{cm}$ & Loss $=0.05 \mathrm{~dB} /$ ring \\
\hline Length $=10 \mathrm{~cm}$ & Length $=3 \mathrm{~mm}$ (200 rings) \\
\hline Group delay $=1.42 \mathrm{~ns}$ & Group delay $=0.2 \mathrm{~ns}$ \\
\hline Group delay ripple $=45 \mathrm{ps}$ & Group delay ripple $=125 \mathrm{ps}$ \\
\hline Simple design & Needs apodization \\
\hline Roughness reduction & r.r. + individual ring tuning \\
\hline
\end{tabular}

Figure 4 Comparison of silicon waveguides and CROWs (as of Year 3 of this project) as a simple fixed delay line structure with an optical insertion loss budget of $10 \mathrm{~dB}$.

We have therefore studied ways to modify the characteristics of silicon microrings after fabrication. One potential method is based on photo-chemistry as shown in polymer microrings [40]. For silicon microrings, our method is based on field-induced local oxidation of $\mathrm{Si}$ to $\mathrm{SiO}_{2}$ via a chemical reaction near an electrically-biased conducting atomic-force microscope tip [41], [42]. For convenience, we used an atomic force microscope available in our lab, but no part of our method depends on "atomic forces". It is simply a site-specific oxidation method, with nanoscale lateral and vertical resolution which is simple to implement and introduces no new materials. In fact, similar scanning probe lithography has previously been used to create nanometer-scale oxide tunnel barriers in silicon and oxide electronic devices and in modifying the resonance frequency of a GaAs photonic crystal cavity. This techniques is part of a larger class of scanning probe lithographic methods developed for physical and chemical surface modification [43].

We continue to study and develop both the single-resonator and coupledresonator configurations for quantum light generation using spontaneous fourwave mixing [44]. The resonator-enhanced nonlinearity benefits the generation of entangled photon-pair and heralded single-photon generation using silicon photonics [18], [19], [45]. A single silicon microresonator can be monitored and controlled more easily [46] than a long CROW device and an optimized single silicon microresonator can also be useful for both nonlinear wavelength conversion [27] and photon-pair generation [47] with low pump powers. 
However, the rates of pair generation using SFWM should be compared with the stronger SPDC (second-order nonlinearity) process, which also be achieved at low pump powers in thin-film lithium niobate waveguides [48].

\section{Open-Access Reporting Initiative}

PRAISE: This open-access document is provided in support of our PRAISE

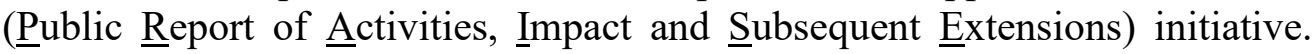
What is it? An open-access document shared with the public which describes the research outcomes of publicly-funded projects. For us, these projects are typically funded by the NSF (National Science Foundation).

\section{References}

[1] S. Mookherjea and A. Yariv, "Optical pulse propagation in the tightbinding approximation," Opt. Express, vol. 9, no. 2, p. 91, Jul. 2001, doi: 10.1364/OE.9.000091.

[2] S. Olivier et al., "Miniband transmission in a photonic crystal coupledresonator optical waveguide," Opt. Lett., vol. 26, no. 13, pp. 1019-1021, 2001.

[3] N. Stefanou and A. Modinos, "Impurity bands in photonic insulators," Phys. Rev. B, vol. 57, no. 19, pp. 12127-12133, May 1998, doi: 10.1103/PhysRevB.57.12127.

[4] T. Mukaiyama, K. Takeda, H. Miyazaki, Y. Jimba, and M. KuwataGonokami, "Tight-binding photonic molecule modes of resonant bispheres," Phys. Rev. Lett., vol. 82, no. 23, pp. 4623-4626, 1999.

[5] W. J. Kim, W. Kuang, and J. D. O'Brien, "Dispersion characteristics of photonic crystal coupled resonator optical waveguides," Opt. Express, vol. 11, pp. 3431-3437, 2003.

[6] S. Mookherjea, D. S. Cohen, and A. Yariv, "Nonlinear dispersion in a coupled-resonator optical waveguide," Opt. Lett., vol. 27, no. 11, p. 933, Jun. 2002, doi: 10.1364/OL.27.000933.

[7] S. Mookherjea and A. Oh, "Effect of disorder on slow light velocity in optical slow-wave structures," Opt. Lett., vol. 32, no. 3, p. 289, Feb. 2007, doi: 10.1364/OL.32.000289.

[8] A. Ciattoni, B. Crosignani, S. Mookherjea, and A. Yariv, "Nonparaxial dark solitons in optical Kerr media," Opt. Lett., vol. 30, no. 5, p. 516, Mar. 2005, doi: 10.1364/OL.30.000516.

[9] B. Crosignani, A. Yariv, and S. Mookherjea, "Nonparaxial spatial solitons and propagation-invariant pattern solutions in optical Kerr media," Opt. Lett., vol. 29, no. 11, p. 1254, Jun. 2004, doi: 10.1364/OL.29.001254. 
[10] J. E. Heebner, R. W. Boyd, and Q.-H. Park, "SCISSOR solitons and other novel propagation effects in microresonator-modified waveguides," J. Opt. Soc. Am. B, vol. 19, pp. 722-731, 2004.

[11] S. Mookherjea and A. Yariv, "Pulse propagation in a coupled resonator optical waveguide to all orders of dispersion," Phys. Rev. E, vol. 65, no. 5, p. 056601, Apr. 2002, doi: 10.1103/PhysRevE.65.056601.

[12] M. L. Cooper et al., "Waveguide dispersion effects in silicon-oninsulator coupled-resonator optical waveguides," Opt. Lett., vol. 35, no. 18, p. 3030, Sep. 2010, doi: 10.1364/OL.35.003030.

[13] S. Mookherjea, "Dispersion characteristics of coupled-resonator optical waveguides," Opt. Lett., vol. 30, no. 18, p. 2406, Sep. 2005, doi: 10.1364/OL.30.002406.

[14] S. Mookherjea and A. Yariv, "Second-harmonic generation with pulses in a coupled-resonator optical waveguide," Phys. Rev. E, vol. 65, no. 2, p. 026607, Jan. 2002, doi: 10.1103/PhysRevE.65.026607.

[15] S. Mookherjea and A. Yariv, "Kerr-stabilized super-resonant modes in coupled-resonator optical waveguides," Phys. Rev. E, vol. 66, no. 4, p. 046610, Oct. 2002, doi: 10.1103/PhysRevE.66.046610.

[16] S. Mookherjea and A. Yariv, "Optical pulse propagation and holographic storage in a coupled-resonator optical waveguide," Phys. Rev. $E$, vol. 64, no. 6, p. 066602 , Nov. 2001, doi: 10.1103/PhysRevE.64.066602.

[17] S. Mookherjea, "Semiconductor coupled-resonator optical waveguide laser," Appl. Phys. Lett., vol. 84, no. 17, pp. 3265-3267, Apr. 2004, doi: 10.1063/1.1719278.

[18] R. Kumar, M. Savanier, J. R. Ong, and S. Mookherjea, "Entanglement measurement of a coupled silicon microring photon pair source," Opt. Express, vol. 23, no. 15, p. 19318, Jul. 2015, doi: 10.1364/OE.23.019318.

[19] R. Kumar, J. R. Ong, M. Savanier, and S. Mookherjea, "Controlling the spectrum of photons generated on a silicon nanophotonic chip," Nat Commun, vol. 5, no. 1, p. 5489, Dec. 2014, doi: 10.1038/ncomms6489.

[20] R. Aguinaldo, Yiran Shen, and S. Mookherjea, "Large Dispersion of Silicon Directional Couplers Obtained via Wideband Microring Parametric Characterization," IEEE Photon. Technol. Lett., vol. 24, no. 14, pp. 1242-1244, Jul. 2012, doi: 10.1109/LPT.2012.2198639.

[21] J. R. Ong, R. Kumar, and S. Mookherjea, "Silicon microring-based wavelength converter with integrated pump and signal suppression," Opt. Lett., vol. 39, no. 15, p. 4439, Aug. 2014, doi: 10.1364/OL.39.004439.

[22] M. L. Cooper and S. Mookherjea, "Numerically-assisted coupledmode theory for silicon waveguide couplers and arrayed waveguides," Opt. Express, vol. 17, no. 3, p. 1583, Feb. 2009, doi: 10.1364/OE.17.001583. 
[23] S. Mookherjea, "Spectral characteristics of coupled resonators," $J$. Opt. Soc. Am. B, vol. 23, no. 6, p. 1137, Jun. 2006, doi:

10.1364/JOSAB.23.001137.

[24] M. L. Cooper et al., "235-ring Coupled-Resonator Optical

Waveguides," in Conference on Lasers and Electro-Optics 2010, San

Jose, California, 2010, p. CTuHH3. doi: 10.1364/CLEO.2010.CTuHH3.

[25] S. Mookherjea and M. A. Schneider, "Avoiding bandwidth collapse in long chains of coupled optical microresonators," Opt. Lett., vol. 36, no. 23, p. 4557, Dec. 2011, doi: 10.1364/OL.36.004557.

[26] M. L. Cooper and S. Mookherjea, "Modeling of Multiband Transmission in Long Silicon Coupled-Resonator Optical Waveguides," IEEE Photon. Technol. Lett., vol. 23, no. 13, pp. 872-874, Jul. 2011, doi: 10.1109/LPT.2011.2141657.

[27] J. R. Ong, R. Kumar, R. Aguinaldo, and S. Mookherjea, "Efficient CW Four-Wave Mixing in Silicon-on-Insulator Micro-Rings With Active Carrier Removal," IEEE Photon. Technol. Lett., vol. 25, no. 17, pp. 1699 1702, Sep. 2013, doi: 10.1109/LPT.2013.2272521.

[28] S. Mookherjea, J. R. Ong, X. Luo, and L. Guo-Qiang, "Electronic control of optical Anderson localization modes," Nature Nanotech, vol. 9, no. 5, pp. 365-371, May 2014, doi: 10.1038/nnano.2014.53.

[29] R. Aguinaldo et al., "Wideband silicon-photonic thermo-optic switch in a wavelength-division multiplexed ring network," Opt. Express, vol. 22, no. 7, p. 8205, Apr. 2014, doi: 10.1364/OE.22.008205.

[30] S. Mookherjea, "Using gain to tune the dispersion relation of coupledresonator optical waveguides," IEEE Photon. Technol. Lett., vol. 18, no. 5, pp. 715-717, Mar. 2006, doi: 10.1109/LPT.2006.871144.

[31] S. Mookherjea and M. A. Schneider, "The nonlinear microring adddrop filter," Opt. Express, vol. 16, no. 19, p. 15130, Sep. 2008, doi: 10.1364/OE.16.015130.

[32] M. L. Cooper, G. Gupta, J. S. Park, M. A. Schneider, I. B. Divliansky, and S. Mookherjea, "Quantitative infrared imaging of silicon-on-insulator microring resonators," Opt. Lett., vol. 35, no. 5, p. 784, Mar. 2010, doi: 10.1364/OL.35.000784.

[33] S. Mookherjea and H. R. Grant, "High dynamic range microscope infrared imaging of silicon nanophotonic devices," Opt. Lett., vol. 37, no. 22, p. 4705, Nov. 2012, doi: 10.1364/OL.37.004705.

[34] M. L. Cooper et al., "Statistics of light transport in 235-ring silicon coupled-resonator optical waveguides," Opt. Express, vol. 18, no. 25, p. 26505, Dec. 2010, doi: 10.1364/OE.18.026505.

[35] J. R. Ong et al., "Low-power continuous-wave four-wave mixing in silicon coupled-resonator optical waveguides," Opt. Lett., vol. 36, no. 15, pp. 2964-2966, 2011. 
[36] F. J. Dyson, "The dynamics of a disordered linear chain," Phys. Rev., vol. 92, pp. 1331-1338, 1953.

[37] N. F. Mott and W. D. Twose, "The theory of impurity conduction," Adv. Phys., vol. 10, pp. 107-163, 1961.

[38] D. C. Herbert and R. Jones, "Localized states in disordered systems," J. Phys. C: Solid St. Phys., vol. 4, pp. 1145-61, 1971.

[39] X. Wang, P. O. Weigel, J. Zhao, M. Ruesing, and S. Mookherjea, "Achieving beyond-100-GHz large-signal modulation bandwidth in hybrid silicon photonics Mach Zehnder modulators using thin film lithium niobate," APL Photonics, vol. 4, no. 9, p. 096101, Sep. 2019, doi: 10.1063/1.5115243.

[40] G. Gupta, Y.-H. Kuo, H. Tazawa, W. H. Steier, A. Stapleton, and J. D. O'Brien, "Analysis and demonstration of coupling control in polymer microring resonators using photobleaching," Appl. Opt., vol. 48, no. 28, p. 5324, Oct. 2009, doi: 10.1364/AO.48.005324.

[41] Y. Shen, I. B. Divliansky, D. N. Basov, and S. Mookherjea, "Electricfield-driven nano-oxidation trimming of silicon microrings and interferometers," Opt. Lett., vol. 36, no. 14, p. 2668, Jul. 2011, doi: 10.1364/OL.36.002668.

[42] Y. Shen, I. B. Divliansky, D. N. Basov, and S. Mookherjea, "Perfect set-and-forget alignment of silicon photonic resonators and interferometers," in Optical Fiber Communication Conference/National Fiber Optic Engineers Conference 2011, Los Angeles, California, 2011, p. PDPC3. doi: 10.1364/OFC.2011.PDPC3.

[43] R. Garcia, R. V. Martinez, and J. Martinez, "Nano-chemistry and scanning probe nanolithographies," Chem. Soc. Rev., vol. 35, no. 1, pp. 29-38, 2006, doi: 10.1039/B501599P.

[44] J. R. Ong and S. Mookherjea, "Quantum light generation on a silicon chip using waveguides and resonators," Opt. Express, vol. 21, no. 4, p. 5171, Feb. 2013, doi: 10.1364/OE.21.005171.

[45] M. Davanço et al., "Telecommunications-band heralded single photons from a silicon nanophotonic chip," Appl. Phys. Lett., vol. 100, no. 26, p. 261104, Jun. 2012, doi: 10.1063/1.4711253.

[46] M. Savanier, R. Kumar, and S. Mookherjea, "Optimizing photon-pair generation electronically using a $p-i-n$ diode incorporated in a silicon microring resonator," Appl. Phys. Lett., vol. 107, no. 13, p. 131101, Sep. 2015, doi: 10.1063/1.4932047.

[47] C. Ma, X. Wang, V. Anant, A. D. Beyer, M. D. Shaw, and S. Mookherjea, "Silicon photonic entangled photon-pair and heralded single photon generation with CAR $>12,000$ and $\mathrm{g}^{\wedge}(2)(0)<0006$," Opt. Express, vol. 25, no. 26, p. 32995, Dec. 2017, doi: 10.1364/OE.25.032995. 
[48] J. Zhao, C. Ma, M. Rüsing, and S. Mookherjea, "High Quality Entangled Photon Pair Generation in Periodically Poled Thin-Film Lithium Niobate Waveguides," Phys. Rev. Lett., vol. 124, no. 16, p. 163603, Apr. 2020, doi: 10.1103/PhysRevLett.124.163603. 